ARTÍCULO

\title{
Escuelas en Territorio Mapuche: desigualdades en el contexto chileno*
}

\author{
Katerin Elizabeth Arias Ortegal (D) \\ Fernando Peña-Cortés' (ID \\ Segundo Quintriqueo Millán' (1) \\ Elías Alejandro Andrade Mansilla' (D)
}

\section{RESUMEN}

$\mathrm{El}$ artículo presenta un estudio exploratorio sobre resultados en la prueba de evaluación del Sistema de Medición de la Calidad de la Educación en comprensión lectora, durante los años 2011 al 2015, en La Araucanía, Chile. Metodológicamente, se describen los resultados obtenidos por una muestra de 22 escuelas emplazadas en comunidades Mapuches. Los resultados dan cuenta que las escuelas municipales rurales, obtienen mejores resultados respecto a las escuelas particulares subvencionadas con una diferencia de 8 a 10 puntos. Esto es relevante, porque en comunidades Mapuches existe una supremacía de escuelas particulares subvencionadas, que sistemáticamente obtienen bajos puntajes, lo que continuaría perpetuando las desigualdades sociales y educativas en el territorio entre Mapuches y no Mapuches.

PALABRAS CLAVE

resultados Sistema de Medición de la Calidad de la Educación; escuelas municipales y particulares subvencionadas; educación rural.

*Agradecimientos al proyecto PROFONDECYT “Dimensión emocional en la relación educativa entre profesor, estudiantes y educador tradicional en la educación intercultural" y al proyecto Fondo Nacional de Desarrollo Científico y Tecnológico (FONDECYT) 1181531. 'Universidad Católica de Temuco, Temuco, Chile. 


\title{
SCHOOLS IN MAPUCHE TERRITORY: INEQUALITIES IN THE CHILEAN CONTEXT
}

\begin{abstract}
The article presents an exploratory study on results in the evaluation tests of the Sistema de Medición de la Calidad de la Educación (Education Quality Measurement System), in reading comprehension, from 2011 to 2015, in La Araucanía, Chile. Methodologically, the results obtained by a sample of 22 schools located in Mapuche communities are described. The results show that rural municipal schools obtain better results compared to subsidized private schools with a difference of 8 to 10 points. This is relevant, because in Mapuche communities there is a supremacy of subsidized private schools, which systematically obtain low scores, which would continue perpetuating social and educational inequalities in the territory between Mapuche and non-Mapuche.
\end{abstract}

\section{KEYWORDS}

Sistema de Medición de la Calidad de la Educación results; municipal and subsidized private schools; rural education.

\section{ESCOLAS EM TERRITÓRIO MAPUCHE: DESIGUALDADES NO CONTEXTO CHILENO}

RESUMO

$\mathrm{O}$ artigo apresenta um estudo exploratório sobre os resultados do teste de avaliação do Sistema de Medición de la Calidad de la Educación (Sistema de Mensuração da Qualidade da Educação), em compreensão de leitura, durante os anos de 2011 a 2015, em La Araucanía, Chile. Metodologicamente, são descritos os resultados obtidos por uma amostra de 22 escolas localizadas em comunidades Mapuches. Os resultados mostram que as escolas municipais rurais obtêm melhores resultados em comparação às escolas privadas subsidiadas, com uma diferença de 8 a 10 pontos. Isso é relevante, pois nas comunidades Mapuches há uma supremacia de escolas privadas subsidiadas, que sistematicamente obtêm pontuações baixas, o que continuaria perpetuando as desigualdades sociais e educacionais no território entre Mapuches e não Mapuches.

\section{PALAVRAS-CHAVE}

resultados do Sistema de Medición de la Calidad de la Educación; escolas municipais e privadas subsidiadas; educação rural. 


\section{INTRODUCCIÓN}

En La Araucanía las escuelas situadas en comunidades indígenas se caracterizan por ser unidocentes, bidocentes o tridocentes, las que se componen por cursos multigrados, impartiendo clases a estudiantes de primer a sexto año de educación básica en una misma sala, o compuestas por cursos combinados de dos niveles. Lo anterior, dependiendo de la cantidad de estudiantes que constituyen los cursos (Williamson, 1993). Las escuelas situadas en comunidades Mapuches rurales o semi-rurales no siempre tienen una cobertura de educación básica completa y están ubicadas fuera del límite urbano. En este contexto, estadísticas del Ministerio de Educación de Chile (MINEDUC), dan cuenta que al año 2016 existe un total de 3.654 escuelas rurales equivalentes a un 30\% de los establecimientos a nivel país, existiendo 12.000 escuelas ubicadas a lo largo del territorio nacional. La matrícula de estudiantes en escuelas rurales asciende a un total de 270.996, equivalente a un 7,6\% de la población total de estudiantes en Chile, correspondiente a 3.548 .736 personas en edad escolar. Para atender a la población escolar en los contextos rurales un total de 26.285 profesores se desempeñan en dichos contextos, equivalente a un $12 \%$ del total país. De este total de profesores, 835 atienden escuelas unidocentes, las cuales se caracterizan porque es solo un profesor quien las dirige y desarrolla el currículum escolar en el establecimiento (www.eligeeducar.cl). Con relación al año escolar 2017, las escuelas situadas en comunidades Mapuches rurales en Chile corresponden al 30\%, equivalente a 3.524 establecimientos rurales y de ellas, el 51,8\% corresponde a las denominadas escuelas multigrado (que atiende entre 1 y 10 estudiantes con varios cursos en una sala). Mientras que el 8,5\% de ellas tiene más de 50 estudiantes. El 21,2\% tiene entre 11 y 20 estudiantes y el 18,5\% entre 21 y 50 estudiantes. De igual modo, en las escuelas rurales durante el período 2017 se atiende a una matrícula de 271.779 estudiantes (www.mineduc.cl).

El área de estudio es la comuna de Padre Las Casas, territorialidad Wenteche (gente del valle), donde constatamos que las escuelas municipales y particulares subvencionadas ubicadas en comunidades Mapuches, en su gran mayoría, son multigrados e incompletas. Otra de sus características, es la diversidad social y cultural de sus estudiantes en las salas de clases. En este sentido, la implementación del sistema educativo monocultural en La Araucanía responde a las necesidades y lógicas del Estado, con relación a mantener las desigualdades sociales entre la población Mapuche y no Mapuche (Mansilla et al., 2016). Para ello, la escolarización de niños indígenas se ha desarrollado teniendo como base un currículum escolar único y monocultural, que desconoce la realidad social, natural, espiritual y cultural en la cual se movilizan sus estudiantes.

En comunidades Mapuches, este sistema educativo se ha constituido en las siguientes fuentes de origen:

- la masificación de la educación a los distintos sectores de la población, desregulándose del aspecto religioso;

- el razonamiento positivista que busca combatir la ignorancia de los sujetos en el ámbito social, político, económico y valórico, para el pro- 
greso social, teniendo a la base los valores universales, en desmedro de los marcos sociales y culturales propios; $\mathrm{y}$

- la conformación de un pensamiento nacionalista (Baudelot y Leclerq, 2008; Donoso et al., 2013).

Estas características del sistema educativo monocultural en territorio Mapuche, han traído como consecuencia una pérdida progresiva de los saberes y conocimientos educativos vernáculos transmitidos en la educación familiar. Es así como las familias Mapuches para evitar la discriminación y prejuicio del no Mapuche, ha relegado la transmisión y uso de los saberes y conocimientos propios al interior de la familia. Esto ha generado en algunas situaciones el ocultamiento y vergüenza étnica a nivel inter e intracomunitario de las personas Mapuches. Desde esta perspectiva, la educación escolar se enmarca en una estructura local con una cierta singularidad de negación y olvido de lo propio, como consecuencia de los tipos de interacciones que se desarrollan con la sociedad hegemónica a través de la escolarización (Boix y Bustos, 2014). En el contexto actual, según Fuica et al. (2014) en Chile, el 79,1\% de las escuelas rurales emplazadas en comunidades indígenas, generalmente son de dependencia municipal. Sin embargo, en el contexto territorial donde se sitúa nuestro objeto de estudio, particularmente, en la comuna de Padre Las Casas, constatamos que la mayor cantidad de establecimientos educacionales ubicados en comunidades indígenas en el sector rural corresponde a escuelas particulares subvencionadas.

En ese sentido, el objetivo del artículo es presentar un estudio exploratorio de los resultados obtenidos por estudiantes de cuarto año de educación básica en la prueba de evaluación Sistema de Medición de la Calidad de la Educación (SIMCE) en comprensión lectora, durante los períodos de 2011 al 2015 en la territorialidad Wenteche, comuna de Padre Las Casas, región de La Araucanía, Chile. Los resultados analizados son de escuelas que se encuentran ubicadas en territorio Mapuche y presentan características sociales, económicas y demográficas similares.

\section{SITUACIÓN DE LA EDUCACIÓN ESCOLAR EN LA ARAUCANÍA}

En Chile la educación escolar, se caracteriza por la desigualdad educativa y la segregación de los estudiantes en el sistema educativo, dependiendo del territorio del cual provienen (Bellei, 2013; Peña-Cortés et al., 2017). La Araucanía, históricamente se ha caracterizado por obtener los más bajos resultados en las evaluaciones estandarizadas en el SIMCE a nivel país (Chile, 2015a). Es lo que observamos en la Tabla 1. Precisamos, que dichas pruebas según el Estado chileno, permiten monitorear la calidad de la educación y los aprendizajes adquiridos por los estudiantes en el sistema educativo escolar.

De acuerdo con los datos observados en la Tabla 1, constatamos diferencias en los puntajes obtenidos a nivel nacional y regional en La Araucanía. Así, los estudiantes en La Araucanía, en la prueba que mide conocimientos disciplinares en la asignatura de lenguaje, en el año 2011 obtuvieron en promedio 14,5 puntos menos que a nivel nacional. Esta diferencia se acrecienta aún más en el año 2012, donde 
Tabla 1 - Puntajes SIMCE a nivel nacional/La Araucanía.

\begin{tabular}{l|c|c|c}
\hline Asignatura & Año & $\begin{array}{c}\text { Puntaje a nivel } \\
\text { nacional }\end{array}$ & $\begin{array}{c}\text { Puntaje escuelas rurales } \\
\text { de La Araucanía }\end{array}$ \\
\hline Lenguaje & 2011 & 267 & 252,5 \\
\hline Matemáticas & 2011 & 259 & 228,2 \\
\hline Lenguaje & 2012 & 267 & 246,9 \\
\hline Matemáticas & 2012 & 261 & 224,6 \\
\hline
\end{tabular}

Fuente: Basado en datos de la Agencia Nacional de Calidad de la Educación, en línea (Chile, 2016c).

Elaboración de los autores.

los estudiantes obtienen una diferencia de 20,1 puntos menos que a nivel nacional. Respecto, a los resultados obtenidos en matemáticas estas diferencias en los puntajes se acrecientan aún más. De tal modo, que los estudiantes de La Araucanía, continúan alcanzando menos puntajes respecto al nivel nacional. Es así como, en el año 2011, la diferencia es de 30,8 puntos menos en La Araucanía. En tanto que, en 2012 la diferencia se acrecienta aún más, donde los estudiantes obtienen una diferencia de 36,4 puntos. En base a los datos, podemos constatar que existen brechas educativas entre escuelas situadas en La Araucanía respecto a escuelas a nivel nacional. En este mismo sentido, hemos podido constatar que a nivel intra-regional, también existen brechas educativas y diferencias entre escuelas situadas en contexto rural y urbano. Asimismo, existen diferencias entre la calidad de los aprendizajes de los estudiantes por género, etnia Mapuche y no Mapuche del contexto rural y urbano, donde aumenta la estratificación social y económica dentro del propio territorio, afectando principalmente al espacio rural y a la población Mapuche (Fernández y Hauri, 2016). En ese contexto, sostenemos que la desigualdad social y la baja calidad en la educación en el contexto territorial de La Araucanía, ha incidido en los resultados de las evaluaciones SIMCE.

En esa misma perspectiva, estudios llevados a cabo por la Universidad de la Frontera a través de su Observatorio Económico Social (OES) durante el período 2013 a 2015, sostienen que los resultados obtenidos en la evaluación SIMCE en La Araucanía, son sistemáticamente bajos. Estas diferencias son mayores cuando los resultados se comparan con escuelas urbanas, principalmente en las evaluaciones de lectura, ciencias naturales y matemáticas. Asimismo, se constata que los estudiantes indígenas sistemáticamente obtienen los menores resultados en las evaluaciones estandarizadas, respecto a los no indígenas. Finalmente, este estudio concluye que, a mayor ingreso socioeconómico de las familias, mayores son los puntajes obtenidos por los estudiantes. En esta perspectiva, sostenemos que los mejores resultados se conjugan con un mayor nivel adquisitivo y accesibilidad de las familias a redes sociales, internet y tecnología. Por lo tanto, esto genera brechas en los aprendizajes, en donde los estudiantes que tienen acceso a mayor tecnología, obtienen mejores logros en las pruebas SIMCE. Es importante recalcar que estas tecnologías y acceso a internet están presentes principalmente en los hogares urbanos, lo que se correlacionaría con los puntajes obtenidos en las evaluaciones SIMCE. 
En esa misma perspectiva, al analizar las estadísticas de la Encuesta de Caracterización Socioeconómica Nacional (CASEN), respecto de la distribución $\mathrm{y}$ asistencia de estudiantes indígenas y no indígenas al sistema educativo escolar, lo que hipotéticamente, podría explicar las diferencias del éxito escolar y educativo entre estudiantes indígenas y no indígenas en la educación escolar. Podemos mencionar que, en educación parvularia no existen diferencias significativas entre estudiantes indígenas y no indígenas que asisten a la educación preescolar, lo que nos permite afirmar que la distribución de estudiantes es equitativa. No obstante, en educación básica comienzan a aparecer diferencias entre 1 a 7 puntos, respecto a los tipos de establecimientos que accede la población indígena y no indígena, donde la asistencia de los estudiantes a la escuela varía según su dependencia municipal, particular subvencionada o particular pagada. Es lo que observamos en la siguiente Tabla 2.

Tabla 2 - Escolarización población indígena en edad escolar 0 a 13 años.

\begin{tabular}{l|c|c}
\hline Nivel educacional & Indígenas & No indígenas \\
\hline Educación parvularia & 51,6 & 51,2 \\
\hline Educación básica municipal & 43 & 38,2 \\
\hline Educación básica particular subvencionada & 54,7 & 53,3 \\
\hline Educación básica particular pagada & 2,1 & 8,2 \\
\hline
\end{tabular}

Fuente: Basado en base a datos de CASEN (Chile, 2017).

Elaboración de los autores.

De acuerdo con los datos presentados en la Tabla 2, constatamos que los estudiantes indígenas asisten, principalmente a establecimientos particulares subvencionados y/o municipales, teniendo una baja presencia en escuelas particulares pagadas (Chile,2017). En tanto que los estudiantes no indígenas, asisten en mayor medida a establecimientos particulares subvencionados y pagados, respecto a estudiantes indígenas. Estos últimos colegios, son los que obtienen, sistemáticamente, mejores resultados en las evaluaciones estandarizadas, lo que podría explicar un mejor nivel educativo de dichos estudiantes, quienes tienen una mayor cobertura curricular y, por ende, tendrían un mayor éxito escolar y educativo. El éxito escolar, refiere a la adquisición y dominio de conocimientos, saberes y competencias, según los programas escolares. Mientras que el éxito educativo, refiere al desarrollo global y óptimo de la persona, considerando las dimensiones de su ser a nivel cognitivo, espiritual, afectivo, físico y social (Arias-Ortega, 2019).

En esa misma perspectiva, constatamos que históricamente, en distintos contextos territoriales que han sido objeto de colonización, han existido diferencias entre la escolarización alcanzada por estudiantes indígenas y no indígenas, la cual es mayor para los últimos (Bishop et al., 2009; Mampaey y Zanoni, 2015; Sarangapani, 2014). En la actualidad, a nivel internacional y nacional, se observan diferencias progresivas respecto a esta realidad histórica, donde los estudiantes indígenas progresivamente han ido alcanzando mayores niveles educativos, sin 
embargo, continúan prevaleciendo diferencias significativas entre la escolarización alcanzada por estudiantes indígenas y no indígenas (Ortiz y Arias-Ortega, 2019). Es así como, en los primeros niveles educativos desde educación parvularia hasta educación media se ha ido nivelando la escolarización entre indígenas y no indígenas, producto de la obligatoriedad de la educación escolar. Sin embargo, aún prevalecen brechas educativas en esos niveles, que se acrecientan en la educación superior, donde podemos evidenciar diferencias significativas entre estudiantes indígenas y no indígenas (Tabla 3).

Tabla 3 - Niveles de escolarización población indígena 18 años o más.

\begin{tabular}{l|c|c}
\hline Nivel educacional & Indígenas \% & No Indígenas \% \\
\hline Sin educación formal & 2,8 & 2,3 \\
\hline Educación básica incompleta & 17 & 11,5 \\
\hline Educación básica completa & 12,5 & 10,1 \\
\hline Educación media incompleta & 12,2 & 12,3 \\
\hline Educación media completa & 30,8 & 30,7 \\
\hline Educación superior incompleta & 11,2 & 12,1 \\
\hline Educación superior completa & 12,3 & 20,5 \\
\hline
\end{tabular}

Fuente: Basado en base a datos de la CASEN (Chile, 2017).

Elaboración de los autores.

De acuerdo con los datos observados en la Tabla 3, evidenciamos diferencias entre los niveles de escolarización entre estudiantes indígenas y no indígenas. Por ejemplo, respecto a los niveles de educación formal en la población de indígenas un 2,8\% no tiene educación formal versus un 2,3\% de la población no indígena. De esta manera, existe una variación del $5 \%$, donde la población indígena obtiene un mayor nivel de desescolarización. Con relación a los niveles de educación básica incompleta, observamos que un porcentaje mayor de población indígena, equivalente a un $17 \%$, no ha terminado su educación básica. Al comparar este mismo nivel educativo con la población no indígena, constatamos que existen diferencias significativas de 6 puntos, a favor de la población no indígena, la que alcanza un mayor nivel de escolarización. En este mismo sentido, respecto al nivel de educación media incompleta y educación media completa, observamos niveles similares de escolarización. No obstante, las brechas educativas entre población indígena y no indígena se acrecientan en los niveles de educación superior, donde es la población indígena la que alcanza menores niveles educativos. Es así como solo un 12,3\% de la población indígena tiene educación superior completa a diferencia de un 20,5\% de la población no indígena. Sostenemos que estas brechas educativas constituyen aspectos que podrían aumentar las desigualdades sociales y económicas entre ambos grupos, viéndose la población indígena desfavorecida, lo que se vería reflejado en su territorio, aumentando la reproducción de la pobreza en territorio indígena.

En suma, al revisar las estadísticas actuales de niveles de escolarización de la población indígena y no indígena, constatamos que la desigualdad educativa 
ha afectado a la población indígena en la actualidad. Así, son los estudiantes indígenas quienes obtienen un menor nivel educacional. Precisamos que esta realidad de desigualdad entre indígenas y no indígenas en el contexto educativo escolar chileno, no es reciente, sino más bien es una constante histórica, que ha sido cuestionada por los propios indígenas, al menos desde 1925 (Arias-Ortega, 2019). Por ejemplo, en 1925 intelectuales indígenas demandaban una educación escolar con pertinencia social, cultural y lingüística que les permitiese, por una parte, acceder al conocimiento sobre la aritmética y el lenguaje desde el conocimiento escolar occidental, como una estrategia para defender su territorio. Y, por otra parte, revitalizar y fortalecer sus saberes y conocimientos educativos propios en la educación escolar, para reforzar su identidad social y cultural, desde un enfoque de prácticas pedagógicas contextualizadas a la realidad en la que se sitúa la escuela, para aportar a la resolución de problemas propios de la comunidad (Arias-Ortega, 2019). De este modo, las brechas educativas son factores que sostienen las desigualdades estructurales en la sociedad y en el territorio, aumentando la estratificación y segregación entre indígenas y no indígenas a nivel intrarregional. Así, el contexto escolar parece no ser capaz de ofrecer a los indígenas, las condiciones efectivas para finalizar con éxito los procesos de escolarización. En ese contexto, exploraremos cómo esta situación se observa en La Araucanía, en la territorialidad Wenteche, específicamente en la comuna de Padre Las Casas, considerando escuelas rurales y urbanas ubicadas en comunidades Mapuches, de dependencia municipal y particular subvencionada.

\section{METODOLOGÍA}

La metodología empleada es la investigación educativa, que se basa en la complementariedad metodológica para construir nuevos conocimientos con relación a las problemáticas educativas en contexto indígena e intercultural, sustentada en un enfoque multimétodo (Bisquerra, 2004; Ruiz, 2008). Para una mejor visualización del análisis descriptivo y comparativo de los resultados obtenidos en las pruebas SIMCE en la comuna de Padre Las Casas, se elaboró una cartografía con información espacial, que presenta la ubicación de las escuelas rurales que participaron en el estudio.

\section{DESCRIPCIÓN SOCIO DEMOGRÁFICA DEL ÁREA DE ESTUDIO}

Desde una mirada geográfica territorial, la región de La Araucanía es un "sistema territorial compuesto por paisajes, que han sido configurados, por las condiciones físico-naturales del espacio geográfico" (Peña-Cortés et al., 2016, p. 199). Es un territorio que posee sus propias especificidades marcadas por una historia étnico-cultural significativa que se ha construido mediante los procesos de colonización, el cual se caracterizó por fragmentar el territorio para segregar a la población Mapuche, distribuyéndolo en los territorios con menor producción agrícola (Peña-Cortés et al., 2016). Este proceso trajo consigo, en primer lugar, la ubicación del Mapuche en sectores alejados del territorio central, como una forma 
de aislarlos e invisibilizarlos en la sociedad. En segundo lugar, esto tuvo como consecuencia, la omisión de los saberes y conocimientos indígenas en el territorio. $\mathrm{Y}$, en tercer lugar, esto da cuenta de los elementos constitutivos de La Araucanía, como lo es su fuerte centralización regional.

Esta región según los estadísticos de la CASEN (Chile, 2017), está compuesta por un total de 957.224 personas. La población de La Araucanía equivale al 5,4\% del total país. De ellas, $465.131(48,6 \%)$ son hombres y 492.093 (51,4\%), mujeres. En este contexto, la comuna de Padre Las Casas, de acuerdo con el Censo del año 2002 alcanzó a 58.795 habitantes, dicha población al año 2015 aumentó a 86.913 habitantes, lo cual indica una variación censal de un 47,82\% según el Reporte Estadístico Comunal (Chile, 2015b). Del total de población en la comuna de Padre Las Casas, 23.922 personas se auto-reconocen como Mapuches, lo que es equivalente a un 40,69\% de la población (Chile, 2016b).

La Araucanía, particularmente, la comuna de Padre Las Casas como área de estudio, históricamente, se ha caracterizado por mantener relaciones interculturales con los no Mapuches, las que han surgido de una manera estereotipada, prejuiciada y mediante eventos de discriminación hacia los habitantes Mapuches (Merino et al., 2009). La comuna de Padre Las Casas se conforma por las siguientes subterritorialidades: Molco, Nirrimapu, Maquehue, Aillacara, Collahue, Roble Huacho, Metrenco, Millahueco y Truf-Truf (Chile,2015b). Además, la comuna se caracteriza por el amplio territorio indígena, que evidencia una intervención y castellanización en la designación de los distintos territorios. Esto implica una descontextualización y desconocimiento de los saberes y conocimientos propios de dichos territorios y de su toponimia étnica, lo que incide en el desconocimiento del propio territorio, por los estudiantes (Quintriqueo et al., 2015).

Geográficamente, la comuna de Padre Las Casas se localiza en la Región de La Araucanía, la que se enmarca entre los $37^{\circ} 35^{\prime}$ y $39^{\circ} 37^{\prime}$ de Latitud Sur y desde los 7050' de Longitud Oeste hasta el Océano Pacífico (Chile, 2014). Territorialmente, la comuna se emplaza en la provincia de Cautín, y posee una superficie de $463,1 \mathrm{~km}^{2}$, que corresponde al $2,17 \%$ de la superficie provincial y al $1,25 \%$ de la superficie regional. La comuna limita al Norte con la comuna de Temuco, al Oeste con la comuna de Nueva Imperial, al Sur con la comuna de Freire y al Este con la comuna de Vilcún (Mapa 1).

De acuerdo con la Mapa 1, observamos que, en el área de estudio se encuentra una gran cantidad de Títulos de Merced, los que se constituyen en documentos que acreditan la propiedad sobre el territorio Mapuche, una vez que el Estado chileno concluyó el proceso de ocupación militar de La Araucanía (Chile, 2003).

\section{CONTEXTO Y PARTICIPANTES}

El contexto de investigación, corresponde a un total de 22 escuelas de educación básica situadas en la comuna de Padre Las Casas, región de La Araucanía, territorialidad Wenteche. En el contexto rural, la muestra se compone por 11 escuelas de dependencia municipal, de las cuales 7 de ellas, se ubican en el grupo socioeconómico de nivel bajo y 4 en el nivel medio-bajo. En el contexto urbano se 


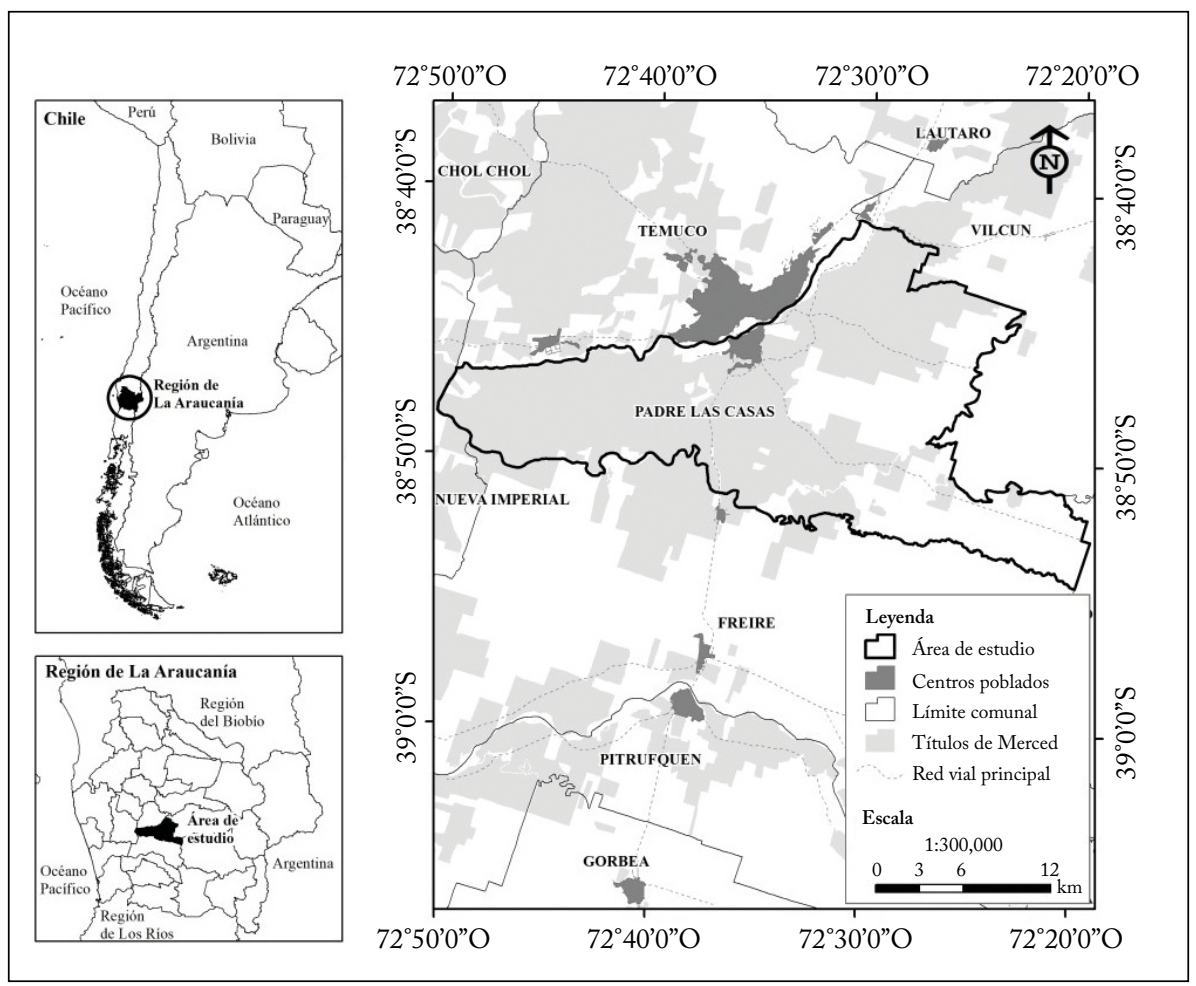

Mapa 1 - Área de estudio comuna de Padre Las Casas, región de La Araucanía, Chile.

Elaboración de los autores.

consideran 11 escuelas de dependencia particular-subvencionado. Para ello, hemos resguardado la pertinencia de las escuelas de la muestra, las cuales pertenezcan a los mismos grupos socioeconómicos del medio rural y que hayan rendido la evaluación SIMCE en el período 2011 a 2015 de manera sistemática. La muestra del estudio se determinó en base a los siguientes criterios:

- la población Mapuche que habita estos territorios pertenece al pueblo Mapuche mayoritario con 1.745.147 habitantes, lo que representa un $79,8 \%$ respecto al total de la población indígena a nivel nacional, correspondiente a 2.185.792 habitantes (Chile, 2017). De igual modo, se constituye en la segunda región con mayor presencia de población indígena, equivalente a un 34,3\%;

- la región de La Araucanía presenta la mayor cantidad de población hablante del mapunzugun a nivel país, cuyo conocimiento se encuentra entre quienes tienen 60 años o más;

- en el ámbito educativo la región se ha caracterizado por obtener bajos resultados en las pruebas estandarizadas del SIMCE, ubicándola en los últimos lugares respecto al resto del país, situación que podría estar ligada a la descontextualización curricular (Chile, 2013); 
- presenta un retraso de más de 100 años en indicadores educativos; y

- la región presenta un nivel de analfabetismo de un 6,9\% frente al 3,5\% a nivel nacional (Peña-Cortés et al., 2017).

De las escuelas seleccionadas, el nivel escolar y económico de los padres en la comuna, se caracteriza por ubicarse en un nivel socioeconómico bajo y medio bajo. Respecto al sector socioeconómico bajo, el nivel educativo de los padres de familia fluctúa entre un promedio de escolarización formal de 9 años. El ingreso económico del nivel bajo, tiene una remuneración máxima de \$250.000 mensuales por hogar. Estas características hacen que la comuna de Padre Las Casas, se caracterice por tener estudiantes entre un $81,01 \%$ a $100 \%$, en condición de vulnerabilidad social (Chile, 2016d). En lo concerniente al sector socioeconómico medio-bajo, el nivel educativo de los padres de familia es de 10 a 11 años de escolaridad, con un ingreso entre $\$ 250.001$ a $\$ 400.000$, mensual. En este contexto, según los datos del MINEDUC (2016), entre el 62,01\% al 81\% de los estudiantes de la comuna de Padre Las Casas se encuentran en condición de vulnerabilidad social. Realidad que es característica de La Araucanía, como la más pobre a nivel nacional (Tabla 4).

Tabla 4 - Regiones con mayor nivel de pobreza a nivel nacional.

\begin{tabular}{l|c|c}
\hline Región & Personas & Porcentaje \\
\hline La Araucanía & 171.368 & 17,2 \\
\hline Nuble & 74.283 & 16,1 \\
\hline Maule & 133.191 & 12,7 \\
\hline Total & 378.842 & \\
\hline
\end{tabular}

Fuente: Basado en base a datos de CASEN (Chile, 2017).

Elaboración de los autores.

En ese sentido, la región se caracteriza por ser el territorio donde se registran los mayores porcentajes de pobreza e indigencia a nivel nacional, donde se agrega, además, la pobreza multidimensional (Rojas-Maturana y Peña-Cortés, 2015). Dicha pobreza, se caracteriza porque no permite a las familias acceder a niveles básicos de bienestar, como salud, vivienda, empleo y educación (Tabla 5).

Tabla 5 - Regiones con mayor nivel de pobreza multidimensional a nivel nacional.

\begin{tabular}{l|c|c}
\hline Región & Personas & Porcentaje \\
\hline La Araucanía & 273.995 & 28,5 \\
\hline Los Lagos & 218.227 & 25,5 \\
\hline Tarapacá & 81.011 & 24,9 \\
\hline Total & 573.233 & \\
\hline
\end{tabular}

Fuente: Basado en base a datos de CASEN (Chile, 2017).

Elaboración de los autores. 
En suma, los datos de la Tabla 4 y 5, nos permite constatar la desigualdad económica y social en la cual, históricamente se ha ubicado La Araucanía, respecto al resto del país. Cabe señalar, que estos resultados son consecuencia del legado colonial y poscolonial que contribuyó sistemáticamente en el empobrecimiento de la población indígena a nivel país, a través de la expropiación de su territorio y la segregación a terrenos con baja productividad agrícola. Asimismo, esto trajo consigo un sistema educativo monocultural que prescinde de los saberes y conocimientos de los pueblos indígenas, el que ha buscado la homogeneización social y cultural del Mapuche a la sociedad nacional, desarraigando de sus marcos de referencia propios (Quintriqueo et al., 2016). Además, esta pobreza también se podría explicar, producto que las grandes empresas que operan en la región, tributan a nivel central en Santiago de Chile, con lo cual deja a La Araucanía desprovista de recursos para financiar la educación y la generación de empresas y emprendimientos locales, aportando al desarrollo local y regional, y, por ende, contribuiría a las familias y comunidades del territorio, permitiéndoles una mayor generación de empleos y recursos económicos. Sin embargo, es una realidad que no sucede en el contexto local.

\section{PROCEDIMIENTOS DE ANÁLISIS DE LA INFORMACIÓN}

El procedimiento de análisis de la información, consistió en organizar la base de datos de la siguiente manera:

- se organizaron las escuelas rurales municipales, de orden creciente y en relación a los puntajes SIMCE obtenidos en los últimos cinco años;

- se organizaron las escuelas urbanas particulares-subvencionadas, de acuerdo a los puntajes obtenidos en las pruebas SIMCE durante los últimos cinco años;

- se mapeó la distribución de las escuelas en la comuna, identificando cuáles de ellas pertenecen a comunidades indígenas.

Para ello, la cartografía se elaboró a partir de la recopilación de información espacial vectorial en formato shape de instituciones públicas, principalmente del MINEDUC, Infraestructura de Datos Espaciales de La Araucanía (IDE Chile), y de la Corporación Nacional de Desarrollo Indígena (CONADI). La información espacial, fue procesada en el software ArcGis 10.1, datum WGS 84, huso 18, zona Sur. A partir de esta agregación de capas de información fue posible evidenciar la distribución de establecimientos educacionales en la comuna, haciendo la distinción según dependencia:

- escuelas particular-subvencionadas; y

- escuelas municipales.

Finalmente, en ambas dependencias se identificó su localización espacial, entendida como rural y/o urbano, evidenciando que la mayor cantidad de establecimientos rurales tanto de escuelas particular-subvencionados como escuelas 
municipales se encuentran localizadas sobre Títulos de Merced, es decir, sobre territorio de comunidades Mapuches de la territorialidad Wenteche;

- cada uno de los establecimientos se le calculó la variación obtenida en su puntaje SIMCE de los últimos cinco años;

- se graficaron esas variaciones, de acuerdo a la dependencia administrativa de la escuela; $y$

- se describieron y contrastaron los resultados obtenidos por grupos.

Un primer grupo se obtuvo de las escuelas municipales rurales y un segundo grupo de escuelas particulares subvencionadas urbanas, para develar si en un mismo contexto territorial existen diferencias significativas. Es preciso recalcar, que esta base de datos se construyó a partir de los resultados publicados en www.simce.cl, donde se individualizó el contexto de la escuela, puntaje obtenido y grupo socioeconómico al que pertenecen. A continuación, la Mapa 2 presenta la distribución de establecimientos educacionales de la comuna de Padre Las Casas, que participan en el estudio.

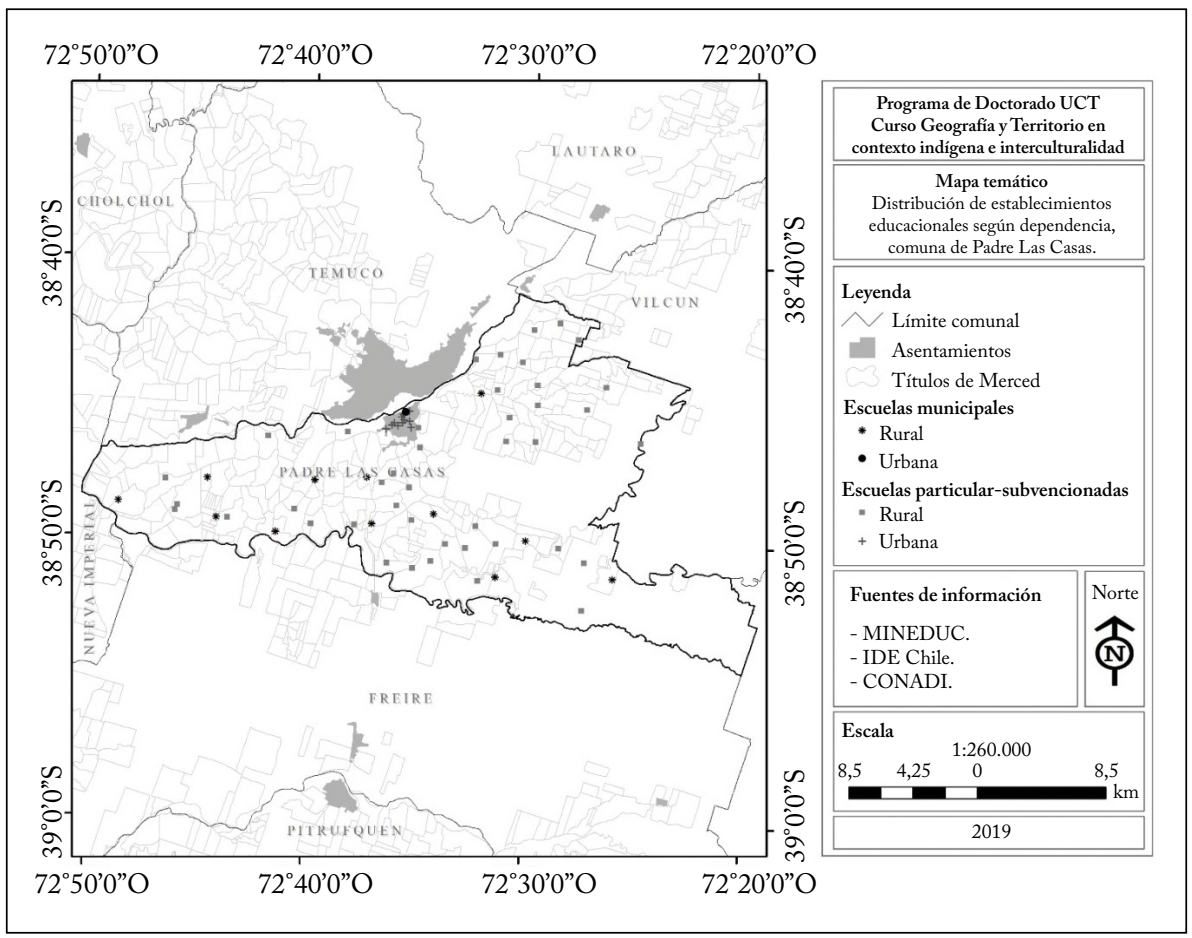

Mapa 2 - Distribución de establecimientos educacionales, comuna de Padre Las Casas.

Elaboración de los autores.

En el mapa de la Mapa 2 se visualiza la distribución de establecimientos educacionales en la territorialidad Wenteche, donde constatamos que en las comunidades Mapuches de la comuna de Padre Las Casas, existe una predominancia de escuelas particulares-subvencionadas, las cuales son propiedad de sostenedores particulares. Sin embargo, también se observa una gran cantidad de escuelas municipales en comuni- 
dades Mapuches en el sector rural. En tanto, en el contexto urbano la predominancia de las escuelas continúa siendo de establecimientos particulares- subvencionados, encontrándose solo una escuela municipal en el área urbana. Desde esta perspectiva, se observa que la oferta educativa en la comuna es variada en las comunidades Mapuches, teniendo una alta presencia de escuelas particulares subvencionadas y municipales en el territorio, constituyéndose en un factor más de segregación social en el territorio. Esto, porque los establecimientos que históricamente han obtenido los más bajos resultados en las evaluaciones estandarizadas, corresponden a establecimientos distribuidos en sectores rurales. Lo anterior, da cuenta que la segregación e inequidad del sistema educativo se manifiesta tanto en el aspecto curricular como en la infraestructura, en la inversión de recursos económicos, didácticos, humanos y en la calidad de los docentes. De esta manera, la segregación se presenta de manera explícita e implícita en el territorio (Bellei, 2013), donde las comunas con mayores ingresos son aquellas que obtienen mejores resultados e inversamente, las comunas con menores ingresos obtienen los más bajos resultados.

\section{RESULTADOS}

Los resultados de la exploración y descripción de los resultados SIMCE obtenidos por escuelas municipales y particulares-subvencionadas rurales en territorio Mapuche, durante los últimos cinco años (período 2011-2015), dan cuenta que existen diferencias significativas entre los puntajes obtenidos por las escuelas municipales del sector rural (MSR), respecto de las escuelas particulares-subvencionadas del sector rural (PSR), tal como se observa en el siguiente Gráfico 1.

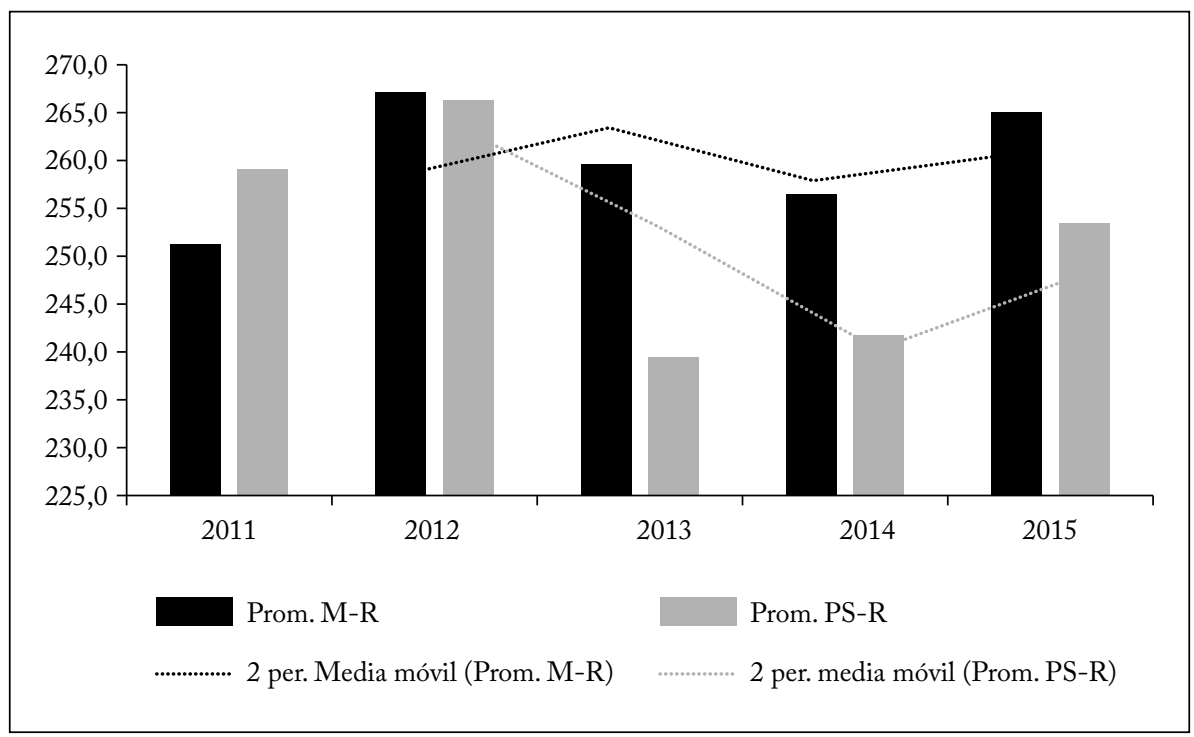

Gráfico 1 - Promedio SIMCE período 2011-2015 escuelas municipales rural/particulares subvencionadas rurales. Prom. M-R: promedio escuela municipal rural; Prom. PS-R: promedio escuela particular subvencionada rural. Elaboración de los autores. 
De acuerdo con el Gráfico 1, observamos que existe una diferencia importante entre los puntajes obtenidos por escuelas municipales rurales, las que obtienen puntajes más altos, respecto a las escuelas particulares subvencionas rurales. Dicha diferencia de puntuación tiene una variación que va de 4 a 25 puntos. Tal como se observa en el período 2013, donde el promedio de las escuelas municipales rurales está por sobre las escuelas particulares-subvencionadas en 20 puntos en el ámbito rural. Estos puntajes, son importantes de discutir, si consideramos que históricamente, las estadísticas han posicionado a las escuelas particulares subvencionadas por sobre las escuelas municipales. Más aún si consideramos que en las comunidades Mapuches predominan las escuelas particulares subvencionadas, las que en el período 2011-2015, estarían obteniendo los peores resultados en el contexto rural. De esta manera, los resultados específicos de la comuna de Padre Las Casas, se contraponen con las estadísticas históricas del MINEDUC. Lo que nos desafía a precisar en la importancia del resguardo de las estadísticas nacionales y cautelando las generalizaciones, que "no necesariamente", explicitan la realidad local.

En ese mismo sentido, al comparar los resultados obtenidos por las escuelas municipales del sector rural y las escuelas particulares subvencionadas del sector urbano, observamos que, aunque comparten características similares en el ámbito económico y educativo, existen diferencias significativas, favoreciendo a las escuelas urbanas, donde no necesariamente, la población que mayormente atiende, corresponda a la población Mapuche. Tal como se presenta en el siguiente Gráfico 2.

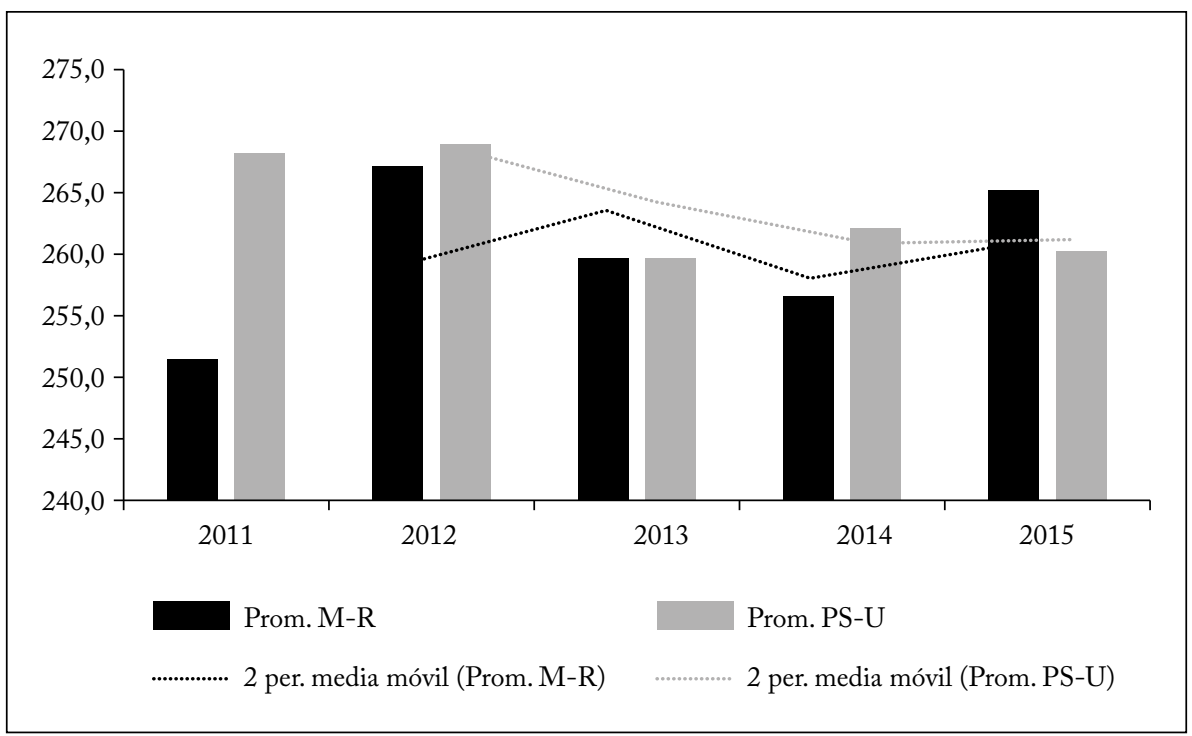

Gráfico 2 - Escuelas municipales rurales con relación a escuelas particulares-subvencionadas urbanas en la comuna de Padre Las Casas.

Prom. M-R: promedio escuela municipal rural; Prom. PS-U: promedio escuela particular subvencionada urbana. Elaboración de los autores. 
El análisis del Gráfico 2, nos permite constatar que existe una variación de los puntajes SIMCE, donde las escuelas particulares-subvencionadas urbanas, obtienen los mayores puntajes con relación a las escuelas municipales rurales, que atiende principalmente población Mapuche en la comuna de Padre Las Casas. Por ejemplo, se observa que en el año 2011 las escuelas particulares-subvencionadas urbanas, obtuvieron alrededor de 10 puntos de diferencia a favor con relación a las escuelas municipales rurales. Observamos que, aun cuando la brecha en el año 2012 disminuye, los puntajes obtenidos por las escuelas particulares-subvencionadas urbanas, continúan siendo mayores. Misma situación ocurre en el año 2013, donde se observan 20 puntos de diferencias a favor de las escuelas particulares-subvencionadas urbanas, mientras que en el año 2014 esto se eleva a 21 puntos de diferencia. Finalmente, en el año 2015 aun cuando la brecha se invierte en 8 puntos de diferencia entre las escuelas municipales rurales con relación a los particulares-subvencionados urbanos, sistemáticamente, son las primeras las que sistemáticamente, obtienen más bajos resultados en el contraste rural/urbano.

En esa misma perspectiva, al triangular los puntajes SIMCE, según dependencia administrativa y ubicación territorial de dichas escuelas, ubicadas en comunidades Mapuches de la territorialidad Wenteche en La Araucanía, constatamos que existen diferencias significativas en los resultados obtenidos (Gráfico 3).

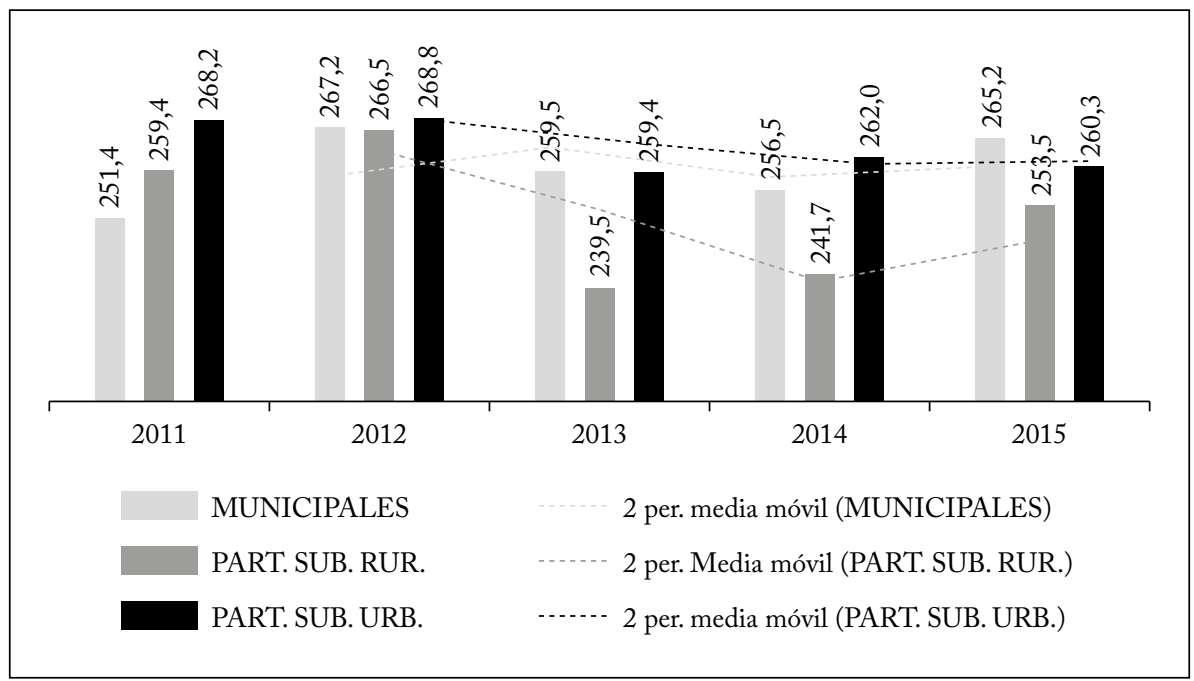

Gráfico 3 - Promedio SIMCE según tipos de establecimiento en territorio Mapuche, período 2011-2015.

PART. SUB. URB.: escuela particular subvencionado emplazada en territorio urbano; PART. SUB. RUR.: escuela particular subvencionado emplazada en territorio rural

Elaboración de los autores.

De acuerdo con el Gráfico 3, constatamos que existe un leve patrón que favorece las evaluaciones SIMCE durante los años 2011-2015, a las escuelas particulares subvencionadas de carácter urbano, por sobre las escuelas municipales y particulares subvencionadas del sector rural. No obstante, al desagregar los datos y comparar a las escuelas municipales y particulares rurales, observamos diferencias interesantes 
de discutir. Así, se observa que las escuelas particulares subvencionadas rurales, son las que obtienen los peores resultados en el contexto rural de La Araucanía, en la territorialidad Wenteche. Aspecto que es problemático, si consideramos que estas escuelas predominan en el territorio Mapuche, lo que podría explicar la persistencia en la reproducción de las brechas educativas y lo que trae como consecuencia, la reproducción de la pobreza en un período de tiempo progresivo. Sin embargo, según los estadísticos, "generalizados" del MINEDUC, históricamente, estos bajos resultados de manera consciente o inconsciente, han sido encubierto o negados. Esto, a través de una estadística generalizada que posiciona a las escuelas particulares subvencionadas por sobre los establecimientos municipales en la región, realidad que no se condice con nuestro estudio exploratorio, desagregando la especificidad propia de la territorialidad Wenteche. Esta realidad plantea el desafío y la responsabilidad de la política educativa, de mayor precisión al analizar los resultados obtenidos en pruebas estandarizadas, lo que podría estar ocultando la crisis del sistema educativo en territorio Mapuche, donde son los estudiantes, los perjudicados a quienes se les estaría entregando una educación escolar de menor calidad respecto a estudiantes no indígenas a nivel nacional y regional.

\section{CONSIDERACIONES FINALES}

Con relación al objetivo del artículo que busca explorar los resultados SIM$\mathrm{CE}$ obtenidos en la territorialidad Wenteche de la comuna de Padre Las Casas durante los años 2011 al 2015, constatamos que, los datos obtenidos en la comuna develan que existen diferencias significativas entre establecimientos de dependencia municipal y particular-subvencionado rural. De esta manera, develamos que las escuelas particulares subvencionadas situadas en comunidades Mapuches rurales son quienes obtienen los más bajos resultados en las evaluaciones estandarizadas. Lo anterior, significa que los estudiantes Mapuches y no Mapuches que asisten a este tipo de establecimientos en la territorialidad Wenteche, no están logrando niveles de aprendizaje adecuados, lo cual les afectará su progresión curricular en el proceso de escolarización (Fernández y Hauri, 2016; Peña-Cortés et al., 2016; Vartanian y Gleason, 1999).

En ese sentido, en contexto de comunidades Mapuches que las escuelas municipales alcancen mejores resultados en la evaluación SIMCE, respecto de las escuelas particulares subvencionadas, podría explicarse, hipotéticamente, por las siguientes razones:

- en las escuelas municipales existe una mayor rigor en la contratación de los profesores, a quienes se les solicita, generalmente, poseer alguna especialización en asignaturas como lenguaje y comunicación, matemáticas, ciencias naturales;

- existirían mayores supervisiones técnicas, didácticas y pedagógicas en el desarrollo de las práctica pedagógicas de los profesores, tanto a nivel municipal como interno en el establecimiento, en función de las supervisiones de clase que realizan los jefes de Unidad Técnico Profesional de las escuelas o sus respectivos directores; y 
- existe una mayor capacitación de los profesores en la escuela, producto de acciones implementadas por la municipalidad.

En lo concerniente a los resultados que obtienen las escuelas particulares subvencionadas ubicadas en comunidades Mapuches, la continuidad de bajos resultados en las pruebas estandarizadas, podría explicarse, hipotéticamente, por:

- una inadecuada gestión pedagógica del quehacer profesional de los docentes en el aula;

- prevalece en el sostenedor el interés económico y de lucro respecto a los beneficios que les pudiese entregar la escuela, en donde lo importante no sería focalizarse en la formación y calidad de la educación que se imparte, sino más bien, generar mayores ganancias;

- no existiría un control o estándares básicos para la contratación del personal docente;

- las escuelas implementan políticas de atracción de estudiantes de sectores urbanos para llevarlos a contextos rurales, para aumentar las matrículas en la escuela; $y$

- padres de familia y comunidades reciben incentivos económicos, por mantener a los estudiantes matriculados en ese tipo de establecimientos.

Lo anterior, es coherente con resultados de investigación de Beltrán (2014), quien sostiene que las escuelas asentadas en contextos de vulnerabilidad social de La Araucanía, Chile, obtienen un logro incipiente de un 89\% en la gestión pedagógica curricular. Esto, referido a la planificación de las acciones, objetivos, procedimientos y métodos educativos utilizados para abordar el currículum escolar en sus prácticas pedagógicas, da cuenta de una baja cobertura curricular con relación al aprendizaje de los estudiantes.

En contextos indígenas, otros factores educativos que podrían incidir en los bajos niveles educativos alcanzados por las escuelas, podrían estar asociados a:

- la monoculturalidad del currículum escolar, sustentado en la hegemonía del conocimiento de corte eurocéntrico occidental, producto de lo cual se desconocen los saberes y conocimientos educativos indígenas en el currículum escolar (Quintriqueo et al., 2016);

- el racismo institucionalizado que niega la incorporación de los conocimientos educativos Mapuches en simetría con los contenidos escolares, lo que promueve prácticas de invisibilización de la episteme indígena en la educación escolar (Arias-Ortega, Quintriqueo y Valdebenito, 2018);

- la baja calidad de la gestión educativa en la mayoría de los establecimientos educacionales municipales de Chile;

- la descontextualización de las prácticas pedagógicas y contenidos escolares en relación a los contenidos educativos propios que poseen los estudiantes (Arias-Ortega, Quintriqueo y Valdebenito, 2018). Sin embargo, sostenemos que dichos conocimientos educativos propios se encuentran presentes en la memoria social de los adultos y ancianos de 
las comunidades Mapuches, como base para implementar un currículum intercultural (Quintriqueo, 2010); y

- el desconocimiento de los profesores, tanto de saberes y conocimientos educativos Mapuches, así como, el bajo dominio de los contenidos disciplinares que inciden directamente en la descontextualización de la educación escolar.

Sin embargo, el análisis de los resultados SIMCE obtenidos en las escuelas municipales y particulares subvencionadas situadas en comunidades Mapuches de La Araucanía, nos permite constatar puntos de convergencia que podrían explicar esos bajos resultados educativos, tanto en las escuelas municipales como en las escuelas particulares subvencionadas. Dichos puntos tienen relación con factores educativos y sociales. Respecto a los factores educativos se relacionan con:

- ambos tipos de escuela a nivel regional obtienen los peores resultados respecto al resto del país; $y$

- ambos tipos de escuela, implementan la política de "atracción" de estudiantes de distintos contextos, sin importar el arraigo territorial, social y cultural del cual provienen.

Esta política de atracción de estudiantes responde a la lógica neoliberal de la educación escolar, en la que se cancela dinero a las familias, para que envíen a sus hijos a colegios que no necesariamente se encuentran ubicados en el territorio (Torres, 2017);

- se trasladan estudiantes de sectores urbanos a colegios rurales, los que, generalmente, son "rechazados o marginados" en el contexto urbano.

Sostenemos que esto podría deberse a una acción "casi planificada", para ofrecer en contexto indígenas una educación de menor calidad, la que termina reproduciendo el desequilibrio territorial a nivel social, económico, territorial y educativo en La Araucanía. Estos aspectos son relevantes si consideramos que los resultados obtenidos han sido similares históricamente en la región, lo cual se continúa reproduciendo a la fecha. De igual manera, estos resultados, podrían ser producto de factores educativos y sociales que estarían incidiendo en dichos resultados. En relación a los factores sociales, estos podrían estar referidos a:

- la colonialidad del poder y del saber instalado en la educación escolar, donde se niega la participación familiar y comunitaria en los procesos educativos escolares, afectando el éxito escolar y educativo de los estudiantes, producto de una educación eurocéntrica occidental que no considera sus saberes y conocimientos propios, como factores que podrían potenciar la educación escolar (Lander, 2000); y

- el aspecto socioeconómico como variable central de disparidad de los resultados escolares en contexto rural, este promueve la reproducción sistemática de la pobreza a través de la educación. 
Según los datos de la encuesta CASEN del 2015, La Araucanía sigue siendo la región más pobre de Chile, donde se estima que la situación de personas cuyo ingreso total mensual es inferior al ingreso mínimo establecido de $\$ 264.000$ pesos chilenos es equivalente a un $23,6 \%$. Así, la pobreza por ingreso no permite satisfacer las necesidades básicas alimentarias y no alimentarias, del grupo familiar en el hogar (Chile, 2015a). También, La Araucanía es la región con mayor pobreza multidimensional a nivel país, alcanzando un total de $29,2 \%$. Esto limita a las personas para alcanzar condiciones adecuadas de vida asociada al acceso a la educación, tener acceso a salud, trabajo y seguridad social, acceso a una vivienda propia y entornos adecuados para el desarrollo social y cultural de los niños. Asimismo, se limita la construcción de redes y cohesión social que sustenten el desarrollo de las personas (Chile, 2015a). Es en este contexto, donde la pobreza estaría incidiendo en los procesos y resultados en los puntajes SIMCE obtenidos por los estudiantes del contexto territorial indígena (Chile, 2016a; Peña-Cortés et al., 2016). Esto podría estar asociado a una inadecuada distribución de los recursos sociales, económicos y laborales que se agrupan principalmente en las grandes metrópolis, desatendiendo los espacios territoriales próximos, como en el caso de la comuna de Padre Las Casas. Dada esa realidad de bajos resultados educativos en La Araucanía, específicamente, en el contexto de comunidades Mapuches, se considera prioritario promover políticas públicas acordes al contexto territorial y geográfico de los establecimientos educacionales. Desde esta mirada, la preocupación debiese focalizarse en disminuir los niveles de segregación territorial, para que consecuentemente con ello, disminuya la segregación escolar.

En consecuencia, los bajos resultados obtenidos en el sistema educativo chileno, ha traído consigo procesos de deslegitimización y demandas de la sociedad civil, con relación a una mejor calidad de educación, tanto en el acceso como en la calidad. Es así como, la deslegitimización del sistema educativo chileno ha dejado en evidencia que una de las principales características del sistema educativo escolar es la reproducción de las desigualdades sociales, la cual se perpetúa con relación a la oferta educativa que se ofrece a los padres, de acuerdo al poder adquisitivo (Donoso y Arias, 2013; Donoso y Schmal, 2009; Espinoza et al., 2011; Farías y Carrasco, 2012). Asimismo, la fragmentación del conocimiento y segmentación territorial de la sociedad civil, tiene directa relación con los recursos económicos que poseen las familias, lo cual limitará la calidad de la educación que reciban sus hijos. De esta manera, dependiendo del grupo socioeconómico en el cual se ubiquen, será el tipo de educación que reciban, siendo los sectores vulnerables correspondientes principalmente al contexto rural e indígena en La Araucanía, quienes se verán menos favorecidos (Donoso y Arias, 2013). Este tipo de desigualdad socioeconómica obliga a los padres a acceder a establecimientos educacionales que no necesariamente, responden a sus necesidades, pero que por factores económicos y de ubicación espacial los obliga a enviar a sus hijos a dicha institución. Si añadimos a lo anterior, la descontextualización territorial, geográfica y monocultural de la escuela, se evidencia que los estudiantes indígenas que acceden a ella, podrían enfrentar situaciones de desventaja, puesto que no se consideran sus saberes y conocimientos educativos propios que portan y que han sido transmitidos, mediante la educación familiar (Quilaqueo y Quintriqueo, 2017). Esto se ve reflejado, 
en la transmisión un solo tipo de conocimiento de tipo eurocéntrico occidental en la escuela, considerado como único y válido, en desmedro de la forma de producción del conocimiento de la población indígena, población rural y conocimientos locales entre otros (Quintriqueo et al., 2014; Tubino, 2013). Dado lo anterior, podríamos suponer que las características del sistema educativo escolar chileno, inciden negativamente en los procesos de enseñanza-aprendizaje que desarrollan los niños Mapuches y no Mapuches en la escuela, lo cual se acrecienta aún más en el contexto rural, profundizándose en los contextos indígenas.

En suma, se hace necesario considerar criterios para una desfragmentación y estudio minucioso de los resultados obtenidos por cada territorialidad urbano/ rural, de manera tal que no se vean afectados por la generalización, lo cual limita tanto la toma de decisiones como las posibles acciones a seguir para mejorar dichos resultados. De esta manera, los datos nos permiten observar que la diferencia entre los puntajes de escuelas municipales sobre las escuelas particulares-subvencionadas, se han mantenido durante los últimos tres años. No obstante, sostenemos que la brecha educativa entre el sector urbano-rural se continúa perpetuando, donde en el área rural se continúan obteniendo los más bajos resultados en las evaluaciones estandarizadas. En esa perspectiva, lo anterior podría explicarse como una consecuencia de la comprensión de una manera global de los resultados SIMCE, sin considerar las diferencias particulares por territorialidad, donde se podría constatar que las comunidades Mapuches, siguen siendo las que sufren violencia implícita y racismo institucionalizado hacia sus niños que acceden a este tipo de educación hegemónica, monocultural y de baja calidad. Aun cuando, en las últimas décadas se han comenzado procesos de visibilización de los saberes y conocimientos educativos Mapuches, y la revitalización de la lengua vernácula y estatus epistémico de sus conocimientos, mediante su incorporación progresiva a la educación escolar. Asimismo, persisten las demandas de reivindicación histórica del territorio ancestral Mapuche, para revertir las desigualdades territoriales entre Mapuches y no Mapuches.

Finalmente, es necesario considerar la dimensión territorial para brindar mejores oportunidades a la educación a los estudiantes Mapuche y no Mapuche. Esto implica, que los problemas del sistema educativo escolar chileno, se analicen desde una mirada territorial. Para ello, es necesario considerar los factores que podrían incidir en las desigualdades educativas en contexto Mapuche, podría ser la distribución de la oferta educativa, la incorporación de conocimientos geográficos y territoriales propios a la educación escolar, para avanzar en prácticas educativas contextualizadas. De esta forma, estos elementos podrían incidir en una mejora significativa de los resultados obtenidos en pruebas estandarizadas como el SIMCE, siempre y cuando se consideren los saberes tradicionales. Sin duda, es necesario seguir profundizando en las particularidades específicas de cada territorialidad, porque la generalización histórica de los bajos resultados atribuidos a escuelas municipales ha contribuido, en la construcción de una representación social negativa de la educación municipal, lo cual ha incidido en los padres de familia al momento de elegir el establecimiento para educar a sus hijos. Situación que en el caso de las escuelas de la muestra en Padre Las Casas no necesariamente es una realidad. 


\section{REFERENCIAS}

ARIAS-ORTEGA, K. Relación educativa entre profesor mentor y educador tradicional en la educación intercultural. 2019. Tesis (Doctorado) - Universidad Católica de Temuco, Chile, 2019.

ARIAS-ORTEGA, K.; QUINTRIQUEO, S.;VALDEBENITO, V.Monoculturalidad en las prácticas pedagógicas en la formación inicial docente en La Araucanía, Chile. Educação e Pesquisa, São Paulo, v. 44, 2018. http://dx.doi.org/10.1590/s1678-4634201711164545

BAUDELOT, C.; LECLERQ, F. Los efectos de la educación. Buenos Aires: Del Estante Editorial, 2008.

BELLEI, C. El estudio de la segregación socioeconómica y académica de la educación chilena. Estudios Pedagógicos, Valdivia, v. 39, n. 1, p. 325-345, 2013. http://dx.doi. org/10.4067/S0718-07052013000100019

BELTRÁN, J. Factores que dificultan la gestión pedagógica curricular de los jefes de unidades técnico pedagógicas. Revista Mexicana de Investigación Educativa, México, v. 19, n. 62, p. 939-961, 2014.

BISHOP, R. et al. Te Kotahitanga: addressing educational disparities facing maori students in New Zealand. Teaching and Teacher Education, Amsterdam, v. 25, n. 30, p. 1-9, 2009. https://doi.org/10.1016/j.tate.2009.01.009

BISQUERRA, R. Metodología de la investigación educativa. Madrid: La Muralla, 2004. BOIX, R.; BUSTOS, A. La enseñanza en las aulas multigrado: una aproximación a las actividades escolares y los recursos didácticos desde la perspectiva del profesorado. Revista Iberoamericana de Evaluación Educativa, Madrid, v. 7, n. 3, p. 29-43, 2014. CHILE. Informe de la Comisión de Verdad y Nuevo trato - 2003. Chile: Gobierno de Chile, 2003.

CHILE. Encuesta de caracterización socioeconómica nacional - CASEN. Ampliando la mirada sobre la pobreza y la desigualdad. Chile: Ministerio de Desarrollo Social, Subsecretaría de Evaluación Social, 2013.

CHILE. Instituto Nacional de Estadística - INE. Compendio estadístico regional La Araucanía informe anual. Chile: Gobierno de Chile, 2014.

CHILE. Encuesta de caracterización socioeconómica nacional-CASEN. Ampliando la mirada sobre la pobreza y la desigualdad. Chile: Ministerio de Desarrollo Social, Subsecretaría de Evaluación Social, 2015a.

CHILE. Instituto Nacional de Estadística - INE. Compendio estadístico regional La Araucanía informe anual. Chile: Gobierno de Chile, 2015b.

CHILE. Encuesta de caracterización socioeconómica nacional - CASEN. Ampliando la mirada sobre la pobreza y la desigualdad. Chile: Ministerio de Desarrollo Social, Subsecretaría de Evaluación Social, 2016a.

CHILE. Estadísticas de población nacional. Reportes de la comuna de Padre Las Casas. Chile: Congreso Nacional de Chile, 2016b. Disponible en: http://reportescomunales. bcn.cl/2015/index.php/Padre_Las_Casas\#Poblaci.C3.B3n_total_a.C3.B1o_2002_y_ proyecci.C3.B3n_de_poblaci.C3.B3n_a.C3.B1o_2015. Acceso en: 7 dic. 2018. 
CHILE. Informe Técnico SIMCE 2014. Chile: Agencia Nacional de Calidad de la Educación, 2016c. Disponible en: http://archivos.agenciaeducacion.cl/ InformeTecnicoSimce_2014.pdf. Acceso en: 7 dic. 2018.

CHILE. Informe Técnico SIMCE 2015. Chile: Ministerio de Educación, Agencia de la Calidad de la Educación, 2016d.

CHILE. Encuesta de caracterización socioeconómica nacional - CASEN. Ampliando la mirada sobre la pobreza y la desigualdad. 2017. Chile: Ministerio de Desarrollo Social, Subsecretaría de Evaluación Social, 2017.

DONOSO, S. et al. Inequidades invisibles en la educación Chilena: brechas entre estudiantes urbanos y rurales en la Prueba PISA de Lectura. Educação \& Sociedade, $n$. 34, n. 125, p. 1.203-1.227,2013.http://dx.doi.org/10.1590/S0101-73302013000400010 DONOSO, S.; ARIAS, O. Desplazamiento cotidiano de estudiantes entre comunas de Chile: evidencia y recomendaciones de política para la nueva institucionalidad de la educación pública en Chile. Revista Latinoamericana de Estudios Urbano Regionales, Santiago, v. 39, n. 116, p. 39-73, 2013. http://dx.doi.org/10.4067/S02507161201300010000

DONOSO, S.; SCHMAL, R. Introducción a la Economía de la Educación: el fenómeno educativo y su connotación económica. Talca: Editorial Universidad de Talca, 2009.

ESPINOZA, L. et al. Limitaciones en el desarrollo de la actividad matemática en la escuela básica: el caso de la aritmética escolar. Estudios Pedagógicos, Valdivia, n. 37, p. 105-125, 2011. http://dx.doi.org/10.4067/S0718-07052011000100006

FARÍAS, M.; CARRASCO, R. Diferencias en resultados académicos entre educación técnico-profesional y humanista-científica en Chile. Calidad en la educación, Santiago, n. 36, p. 87-121, 2012. http://dx.doi.org/10.4067/S0718-45652012000100003

FERNÁNDEZ, M.; HAURI, S. Resultados de aprendizaje en La Araucanía. La brecha de género en el SIMCE y el androcentrismo en el discurso de docentes de lenguaje y matemática. Calidad en la Educación, Santiago, n. 45, p. 54-89, 2016. http://dx.doi. org/10.4067/S0718-45652016000200003

FUICA, P. et al. Habilidades cognitivas, contexto rural y urbano: comparación de perfiles WAIS-IV en jóvenes. Terapia Psicológica, Santiago, n. 32, n. 2, p. 143-152, 2014. http://dx.doi.org/10.4067/S0718-48082014000200007

LANDER, E. La colonialidad del saber: eurocentrismo y ciencias sociales. Perspectivas Latinoamericanas. Buenos Aires: CLACSO, Consejo Latinoamericano de Ciencias Sociales, 2000.

MAMPAEY, J.; ZANONI, P. Reproducing monocultural education: ethnic majority staff's discursive constructions of monocultural school practices. British Journal of Sociology of Education, United Kingdom, v. 37, n. 7, p. 928-946, 2015. https://doi.or $\mathrm{g} / 10.1080 / 01425692.2014 .1001059$

MANSILLA, J. et al. Instalación de la escuela monocultural en la Araucanía, 1883-1910: dispositivos de poder y Sociedad Mapuche. Educação e Pesquisa, São Paulo, v. 42, n. 1, p. 213-228, 2016. http://dx.doi.org/10.1590/S1517-9702201603140562 
MERINO, M. et al. Perceived discrimination amongst indigenous people in Chile. An application of the Australian taxonomy. Journal of Ethnic and Racial Studies, Inglaterra, n. 32, p. 802-822, 2009. https://doi.org/10.1080/01419870802037266

ORTIZ, E.; ARIAS-ORTEGA, K. Ser Mapuche en la universidad: condiciones de ingreso a la formación inicial docente, La Araucanía. Revista Electrónica Educare, Costa Rica, v. 23, n. 1, p. 1-18, 2019. http://dx.doi.org/10.15359/ree.23-1.1

PEÑA-CORTÉS, F. et al. Priorización de territorios para la gestión educativa en la Araucanía (Chile). Revista Perfiles Latinoamericanos, México, v. 25, n. 49, p. 225-249, 2017. http://dx.doi.org/10.18504/p12549-010-2017

PEÑA-CORTÉS, F. et al. Un modelo de retención universitaria de estudiantes de alto rendimiento escolar de la región de La Araucanía: el caso de los estudiantes Mapuches. In: CONFERENCIA SOBRE EL ABANDONO EN LA EDUCACIÓN SUPERIOR, 4., 2016. Medellín. Medellín: Clabes, 2016. p. 1-7.

QUILAQUEO, D.; QUINTRIQUEO, S. Métodos educativos Mapuches: retos de la doble racionalidad educativa. Aportes para un enfoque de educación intercultural. Temuco: Ediciones Universidad Católica de Temuco, 2017.

QUINTRIQUEO, S. Implicancias de un modelo curricular monocultural en contexto Mapuche. Santiago de Chile: Lom, 2010.

QUINTRIQUEO, S. et al. Formación del profesorado en educación intercultural en América Latina. El caso de Chile. Revista Electrónica Interuniversitaria de Formación del Profesorado, Zaragoza, v. 17, n. 2, p. 201-217, 2014. https://doi. org/10.6018/reifop.17.2.198021

QUINTRIQUEO, S.; QUILAQUEO, D.; GUTIÉRREZ, M.; PEÑA-CORTÉS, F. Enseñanza de historia, geografía y ciencias sociales: hacia una perspectiva intercultural. Temuco: Ediciones Universidad Católica de Temuco, 2015.

QUINTRIQUEO, S. et al. Interculturalidad para la formación inicial docente: desafíos para construir un dialogo intercultural. Temuco: Ediciones Universidad Católica de Temuco, 2016.

ROJAS-MATURANA, M.; PEÑA-CORTÉS, F. Saberes ambientales Lafkenche en escuelas de la costa de La Araucanía (Chile). Revista Latinoamericana de Ciencias Sociales Niñez y Juventud, Colombia, n. 13, p. 1.207-1.220, 2015.

RUIZ, G. Reflexiones y definiciones desde la teoría biológica del conocimiento: aprendizaje y competencia en la universidad actual. Estudios Pedagógicos, Valdivia, v. 34, n. 1, p. 199-214, 2008. http://dx.doi.org/10.4067/S0718-07052008000100012

SARANGAPANI, P. Savoir, curricula et méthodes pédagogiques: le cas de l'Inde. Revue Internationale d'Éducation de Sèvres, Sèvres, 2014. Colloque L'Éducation en Asie en 2014: Quels enjeux mondiaux? Disponible en: https://journals.openedition. org/ries/3848\#quotation. Acceso en: 26 oct. 2015.

TORRES, H. La educación intercultural bilingüe en Chile: experiencias cotidianas en las escuelas de la región Mapuche de La Araucanía. 2017. Tesis (Doctorado) Université de Laval, Quebec, Canadá, 2017. 
TUBINO, F. Intercultural practices in Latin American Nation States. Journal of Intercultural Studies, Australia, v. 34, n. 5, p. 604-619, 2013. https://doi.org/10.1080 /07256868.2013.807538

VARTANIAN, T.; GLEASON, P. Do neighborhood conditions affect high school dropout and college graduation rates? Journal of Socio-Economics, Reino Unido, $\mathrm{n}$. 28, n. 1, p. 21-41, 1999. https://doi.org/10.1016/S1053-5357(99)00011-6

WILLIAMSON, G. Escuelas rurales incompletas con cursos multigrado. Experiencias en Chile: revisión bibliográfica. Talca: Universidad de Talca/ Instituto de Investigación y Desarrollo Educacional, 1993. (Documento de Estudio, n. 1).

\section{SOBRE LOS AUTORES}

Katerin Elizabeth Arias Ortega es doctora en educación por la Universidad Católica de Temuco (Chile). Profesora de la misma institución. E-mail:karias@uct.cl

Fernando Peña-Cortés es doctor en ciencias ambientales por la Universidad de Concepción (Chile). Profesor de la Universidad Católica de Temuco (Chile).

E-mail: fpena@uctemuco.cl

Segundo Quintriqueo Millán es doctor en educación por la Universidad de Extremadura (España). Profesor de la Universidad Católica de Temuco (Chile).

E-mail: squintri@uct.cl

Elías Alejandro Andrade Mansilla es estudiante de doctorado en educación por la Universidad Católica de Temuco (Chile). Profesor de la misma institución.

E-mail: elias.andrade.mansilla@gmail.com

Recibido el 27 de marzo de 2019

Aprobado el 12 de agosto de 2019 